\title{
Application of the Recommended Learning Path in the Personalized Adaptive E-learning System
}

\author{
Vija VAGALE $^{1,2}$, Laila NIEDRITE ${ }^{1}$, Svetlana IGNATJEVA ${ }^{2}$ \\ ${ }^{1}$ Faculty of Computing, University of Latvia, Raina Boulv.19, Riga, LV-1586, Latvia \\ ${ }^{2}$ Faculty of Natural Sciences and Mathematics, Daugavpils University, \\ Vienibas 13 St., Daugavpils, LV-5401, Latvia \\ ORCID 0000-0002-5428-6441, 0000-0002-8173-6081, 0000-0002-3608-8409 \\ vija.vagale@gmail.com, laila.niedrite@lu.lv, \\ svetlana.ignatjeva@du.lv
}

\begin{abstract}
The study analyses how the topic sequences (teacher, learner, optimal) proposed in the personalized adaptive e-learning system influence the learning outcomes of the course. Three experiments were organised within the study. The first experiment showed that in most cases higher grades got those students who used the learner topic sequence. That lead to the need for improving the method for obtaining the optimal topic sequence. In the second experiment, the importance and complexity of the course topics was assessed. Next, weights for each topic were calculated based on the grade, importance and complexity of the topic. A recommended learning path development was proposed. The third experiment of the study evaluated the recommended learning path. The results showed that the recommended learning path gave better learning outcomes for two topics when compared to the optimal topic sequence. Future research will focus on the recommended learning path validation using larger sample group.
\end{abstract}

Keywords: personalized adaptive system, e-learning, recommended learning path, topic sequence, graph.

\section{Introduction}

Nowadays learning process is facing new and large challenges. It is often necessary to organise the learning process remotely and shift the emphasis between the parties involved in the learning process. For these reasons, e-learning systems are becoming increasingly important in the learning process. Consequently, many researchers (Durand et al., 2013; Vagale et al., 2018; Aeiad and Meziane, 2019) are focusing on developing a personalized adaptive e-learning system. The personalized adaptive e-learning system provides learners with the opportunity to take control over the own learning process and offers the individually adjusted content based on the characteristics describing the learner, and his needs (Aeiad and Meziane, 2019).

The main role of the learning process has been granted to the learner aiding the learner-/student-centred e-learning (Jurane-Bremane, 2018). "The Standards and 
Guidelines for Quality Assurance in the European Higher Education Area" also emphasise that "higher education requires a fundamental shift in its provision; it requires a more student-centred approach to learning and teaching, embracing flexible learning paths and recognising competences gained outside formal curricula" (WEB, a).

The emphasis of the learner-centred approach is transferred from teaching to learning. Unlike the traditional approach, the learner-centred approach focuses on the student and his learning. The teacher is the "mediator" between the learner and the acquired knowledge. The main task of the learner-centred approach is to help the learner to acquire the learning skill (Jurane-Bremane, 2018). Some of the features of the learnercentred learning approach are (Lea et al., 2003; Attard et al., 2010): (a) reliance on the active work of the learner; (b) the emphasis on deep learning and understanding; (c) higher responsibility and accountability of the learner; (d) increased sense of autonomy in the learner; (e) participation of the learner in the planning of the own learning process.

The aforementioned features indicate that the learner-centred learning approach can be successfully realized in the personalized adaptive system. The use of such a learning approach raises up the question about the management of the learning process of the learner and the use of the recommended learning path within the personalised adaptive elearning system.

In the study, a learner model based personalized adaptive e-learning system (LMPAELS) developed by the authors (Vagale et al., 2020a) was used. In the LMPAELS, three course topic sequences have been realized: the learner topic sequence, the teacher topic sequence and the optimal topic sequence (Vagale and Niedrite, 2016a). Teacher topic sequence is created by the teacher. The optimal topic is based on the highest topic grades and links between topics. Learner topic sequence is formed during the course acquisition by the learner choosing the topics to study based on links between topics. The default topic sequence is a teacher topic sequence, however, the learner can change the selected topic sequence several times.

The study was divided into three stages. In the 1st stage, the use of the three topic sequences proposed in the e-learning system was evaluated. The aim was to determine which of the used topic sequences show the highest learning outcomes. In the 2nd stage, based on the results obtained in the 1st stage, the optimal topic sequence creation method was improved. As a result, an algorithm for obtaining the recommended learning path was developed. The 3rd stage contains the evaluation of the developed recommended learning path creation algorithm.

The results of the first and second stages of the study are presented in the paper (Vagale et al., 2020b). This paper is an extended version of the previous publication by authors (Vagale et al., 2020b) presented in 14th International Baltic Conference on Databases and Information Systems (DB\&IS 2020). The results of all three stages of the study are included in this paper.

Three experiments were performed throughout the study. The first stage of the study includes two experiments and the third experiment was performed in the third stage of the study. In the first experiment, data were gathered and analysed, explaining how the learners used the three topic sequences for the course acquisition that are realised in the system. The grades of every topic depending on the chosen topic sequence were also obtained. The results of this experiment demonstrated that:

a) learners are passive in the choice of topics. In most cases they used the default topic sequence (i.e. teacher topic sequence). $64,1 \%$ of all students used teacher topic sequence, $20,3 \%$ of students used optimal topic sequence and $15,6 \%$ - learner topic 
sequence;

b) in $80 \%$ of the cases, higher topic grades were of those students who used learner topic sequence and $20 \%$ - optimal topic sequence;

c) the grades of the half of the number of students who used the optimal topic sequence were in the same level as of those using teacher topic sequence.

The conclusions of the first experiment promoted the idea of improving the optimal topic sequence method used in LMPAELS.

In the second experiment, the topic acquisition influential parameters were obtained and analysed with a goal to improve the existing optimal topic sequence method and propose a recommended learning path development algorithm. The chosen parameters characterising topics were grade, complexity and importance. The topic grade describes the learner's chosen level of the topic acquisition and it is acquired by testing the learner. The topic complexity characterises the level of the topic acquisition difficulty. The topic importance describes the location of the topic in the course.

In the third experiment, learning process data were obtained and analysed using teacher topic sequence, learner topic sequence, and recommended learning path. Comparison of the topic learning grades was performed in the case of optimal topic sequence and recommended learning path.

In the literature review various personalized and/or adaptive systems that are employing a learning path are analysed. To our knowledge, no previous research has investigated methods of searching the learning path based on the graph theory and are using the following topic characterizing parameters: topic grade, complexity and importance. We found a graph to be a promising tool for the search of the recommended learning path on the grounds that a graph is a mathematical structure consisting of elements with reciprocal relationships or link (Durand et al., 2013). In the graph we are proposing, vertices represent learning objects/topics whereas edges between them are the existing paths between topics. The weights are calculated both for the vertices and edges of the graph. First of all, the weights of the graph vertices are calculated based on the values of three object/topic characterizing parameters: topic grade, complexity (from the learner's and teacher's perspective), and importance (from the learner's and teacher's perspective) ratings. Secondly, the weights of the edges are calculated based on the weights of the adjacent vertices. Finally, the recommended learning path is modelled based on the weights of the graph vertices and edges. The recommended learning path in this paper represents the sequence of all the learning elements (topics) that gains the best course acquisition results.

The key contribution of this work is the developed un validated algorithm for the recommended learning path development that was used for the "Programming Foundations I" course, described in this paper. A significant benefit of the method is the provisional "independence from the teacher" for the learner, at the same time, offering the path for achieving good results and not "letting the learner in the self-flow".

The paper is organised as follows: Section 2 reviews related works. Section 3 describes the development of the optimal topic sequence method and the personalized adaptive system used in the experiment. Section 4 covers the $1^{\text {st }}$ stage of the study by analysis of the use of the topic sequences used in the e-learning system. The algorithm for the recommended learning path development (the $2^{\text {nd }}$ stage) is explained in Section 5. Section 6 provides a discussion and evaluation of the experiment results using the recommended learning path (the $3^{\text {rd }}$ stage). Finally, concluding remarks and future work are drawn in Section 7. 


\section{Related work}

Numerous computing techniques such as neural networks, fuzzy systems and genetic algorithms are used to create learning paths (Nikman, 2017). A learning path is used not only in traditional learning systems with a limited number of learners and learning objects, but also in systems with a large number of learners, courses and learning objects (Durand et al., 2013; Heberle et al., 2014).

Heberle et al. (2014) is using the learning path to address the problems of high dropout rate in the Massive Open Online Course (MOOC) system. Considering the learner's didactic factors (e.g. age, difficulty level), an individualized ontology is created, with the help of which the most suitable learning objects and the learning paths are identified. Based on the obtained ontology, a semantic query is generated, in which the corresponding small open educational resources are selected.

Aeiad and Meziane (Aeiad and Meziane, 2019) describe a personalized adaptive system consisting of three models: the learner model, the knowledge extraction model, and the content delivery model. The modelling of the content of a specific learning subject is done using ontology. Learners are provided with freely accessible online learning resources. The content is adapted using a learning style of learner (visual, auditory, reading/writing, kinaesthetic) and prior knowledge (beginner, intermediate, advanced). Two vectors are used to create the learning content: the learning concept vector and the vector of the XHTML network elements. Then a program is developed for the learner to learn a specific module. The program consists of topics, web links, hours needed to learn each topic, exercises and evaluation. Content adaptation is based on learner feedback - learner responses (5 questions) on satisfaction with the proposed learning process (content offered, learning experience, etc.) and the quality of the content delivered.

Patel et al. (2017) analyses the learning paths commonly used by students. The analysis uses sequence clustering and graph-based process mining on educational data. All student activities are recorded in the event log. The events are selected that tell about the student's interaction with the digital assets and the platform. From the obtained events, Student ID, Activity ID, and Timestamp are selected. Next, a matrix $n \times n$ is created, where $\mathrm{n}$ is the number of activities the student performed. The matrix records how many times students performed actions $\mathrm{i}$ and $\mathrm{j}$. The resulting matrix was used to construct a directed graph. Using cluster analysis, the most commonly used the learning paths were identified in the graph.

Durand et al. (2013) describes a learning design recommendation system that uses a directed graph for a personalized the learning path search. The vertices of the graph describe the learning objects and the edges indicate the links between these objects. For each vertex, a pair of competencies is defined - the competencies needed to master the specific learning object (prerequisite competencies) and the competencies acquired after the acquisition of the particular learning object. The learning system operates with a large amount of the learning objects stored in the distributed database Cassandra. The learning path search is based on the competences that the learner is expected to acquire.

The learning path described in (Durand et al., 2013) is based on prerequisite and acquired competencies. In our algorithm for development of a recommended learning path, competencies are implicitly identified through links between topics. Our proposed algorithm for developing the recommended learning path operates on any number of learning object descriptive parameters. 


\section{Background}

\subsection{Personalized adaptive e-learning system}

A learner model-based personalised adaptive e-learning system LMPAELS, that is based on the open source content management system Moodle, has been used in the research. Three models are used in the system: a learner model, a content model and an adaptation model.

The learner model describes an adult learner and is used for lifelong learning. This model includes following data categories: personal data, personality data, pedagogical data, preference data, device data, system experience, current moment's knowledge, and history data about learning process. A complete description of the learner model is given in the article (Vagale and Niedrite, 2014).

The content model represents the learning course structure and the variety of the resources used in the course. A learning course consists of topics, where each topic consists of learning objects (e.g. a specific concept from the topic to be learned). Each learning object has four parts: topic summary, theory, practice, and evaluation. The evaluation part includes the developed tests based on varying course difficulty level (chosen by the learner). The learning content of varying resource formats depends on the learning style chosen by the learner. A complete description of the content model can be found in (Vagale and Niedrite, 2014).

The LMPAELS system is used for adaptation of (i) the course structure, (ii) the course content, and (iii) the course topic sequence. The course structure in the system adapts depending on the course type (theoretical or practical). The theoretical courses do not anticipate exercises; therefore, the structure of the learning object excludes the practical part. The content adaptation occurs based on the learner's pre-knowledge (yes, no) in the specific course, as well as learning style (visual, aural, read, kinaesthetic, the combination of visual and aural) and the chosen course difficulty level (low, middle, high) (Vagale et al., 2018).

Three kinds of topic sequences have been realised in the system: teacher, learner and optimal (Vagale and Niedrite, 2016a). Topic sequence is a selection of topics arranged based on specified feature. Teacher topic sequence is based on the teacher's experience. The topics in it are arranged corresponding to their order numbers. Learner topic sequence is created by the learner and his choice of studied topics. After the acquisition of the specific topic, the system offers the next topics based on the teacher's indicated links between topics. If the topic $T_{j}$ can be studied after the topic $T_{i}$, then there exists a directed link from topic $T_{i}$ to topic $T_{j}$. Optimal topic sequence is developed using the optimal topic sequence method (see Section 3.2). The management of course topic sequence in the system is described in the previous article (Vagale and Niedrite, 2016b). A complete description of the architecture of personalised adaptive system is given in paper (Vagale et al., 2018) and system implementation is provided in paper (Vagale et al. 2020a).

\subsection{Optimal topic sequence method}

The optimal topic sequence method is based on learners' achievements (grades) in the course, topic sequences used by the learner for course acquisition and links between the topics of the course. The principle of the method is following: in the acquisition of a 
specific course, all the topic sequences used by learners are divided into groups where each group includes equal topic sequences. Then, the mean course acquisition grade is calculated for each topic sequence group. The topic sequence with the highest mean grade is assumed to be the optimal topic sequence of the specific course. In case of multiple topic sequence groups with equal mean grades, the optimal topic sequence is searched within these topic sequences based on the highest topic repetition frequency in the specific topic sequence position and links between topics. When developing optimal topic sequence, the existence of links between the last chosen topic and the selected following topic is checked. Next check searches that the selected topic is not included in the optimal topic sequence before. The complete optimal topic sequence (OTS) development algorithm is give in the article (Vagale and Niedrite, 2016a).

In both experiment the learners studied "Programming Foundations I" course. The course consists of the following ten topics: (1) "C++ program structure. Data output", (2) "Data types. Data input", (3) "Mathematical functions", (4) "Conditional constructions", (5) "User-defined functions", (6) "Parametric functions", (7) "Cyclic constructions", (8) "One-dimensional numeric arrays", (9) "Multi-dimensional numeric arrays", and (10) "Symbolic arrays". The numbers in the brackets represent the topic sequence number and matches the topic sequence offered by the teacher. There are links defined between the topics that indicate which topics can be studied after finishing the selected topic.

Figure 1 represents links between the topics, where the vertices of the graph, represented by numbers, are the topics and edges between them indicate the link using directed arrows. For example, topic "4" can be followed by topics "5" or "7".

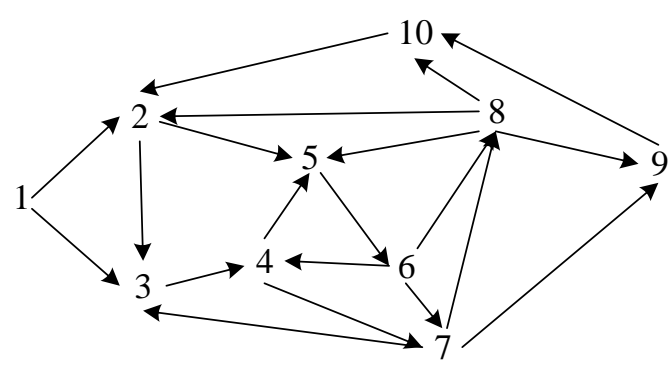

Figure 1. The interconnected course topics graph (Vagale and Niedrite, 2016a).

In the both experiment, OTS $=\{1,3,5,7,8,9,10,2,4,6\}$ was used and it acquisition is described more in the paper (Vagale and Niedrite, 2016a). In the case of a large number of learners, the optimal topic sequence method is working correctly. However, when the number of learners is small, this method does not help creating a better topic sequence. That was proven by the results of the experiment (see Section 4.2). The described optimal topic sequence method is using topic grades and links between topics. The improved method proposed in this paper suggests including additional parameters characterising each topic, like, the complexity and importance. The use of several characterising parameters allows to evaluate the location of each topic within the course in a more objective way. 


\section{Evaluation of the use of topic sequences}

The three experiments that are part of this study were was organised in Daugavpils University (Latvia). The experiment involved first-year students of the professional bachelor study program "Information Technologies". During the study process, students were given the opportunity to study a practical course "Programming Foundations I" in the learner based personalised adaptive e-learning system LMPAELS. The course includes ten mandatory topics (see Section 3.2) in accordance with the study program accredited by the university. Each topic of the course consists of one learning object. After completing the course, each student assessed their learning process by filling in an electronic questionnaire.

This section presents the main results of the two experiments of the first phase of the study. In the first experiment, data on the use of topic sequences (teacher, learner and optimal topic sequence), implemented in the LMPAELS, and the grades of learners in each topic sequence were obtained. In the second experiment, data describing the course topics (parameters' values), such as topic grade, topic complexity, and topic importance, were obtained.

The first experiment took place in 2015/2016 and 2016/2017 with 47 students participating. The second experiment took place in 2019/2020 with 25 students participating. As some students did not complete the course due to personal reasons, only the data of 17 student learning process remained valid. As a result, the study used (a) learning process data of 64 students, such as the topic sequences used in the learning process, the choice of topic sequences, the position of each topic in the learning path, and grades obtained in each topic and (b) the complexity and importance rating for topics by 17 students. The topic grades are on a scale of 1 to 10 . Learners were tested after finishing each topic. A topic is considered passed if the grade in the test is higher than 4 (including).

In the second experiment, after each topic, the learners rated the complexity and importance of the topic on a scale from 1 (lowest) to 10 (highest). The teacher also evaluated the complexity and importance of each topic in order to gain an objective opinion. Table 1 summarizes the arithmetic mean grades of learners, as well as, the arithmetic means of the complexity and importance of each topic from the perspective of the learners, and the teacher. "T" stands for topics, where the index indicates the topic number.

Table 1. Parameters describing the course topic and their arithmetic mean values.

\begin{tabular}{lcccccccccc}
\hline Parameters of topic & $\mathrm{T}_{1}$ & $\mathrm{~T}_{2}$ & $\mathrm{~T}_{3}$ & $\mathrm{~T}_{4}$ & $\mathrm{~T}_{5}$ & $\mathrm{~T}_{6}$ & $\mathrm{~T}_{7}$ & $\mathrm{~T}_{8}$ & $\mathrm{~T}_{9}$ & $\mathrm{~T}_{10}$ \\
\hline Grade & 8.11 & 6.59 & 6.89 & 7.05 & 7.04 & 6.44 & 7.23 & 6.91 & 7.14 & 5.98 \\
Complexity (learner) & 2.77 & 4.59 & 4.77 & 5.18 & 5.05 & 6.09 & 5.59 & 5.91 & 5.05 & 6.18 \\
Complexity (teacher) & 1.00 & 2.00 & 4.00 & 6.00 & 5.00 & 7.00 & 8.00 & 9.00 & 10.00 & 7.00 \\
Importance (learner) & 8.82 & 7.91 & 8.27 & 8.95 & 8.64 & 8.73 & 8.68 & 8.77 & 8.77 & 9.05 \\
Importance (teacher) & 1.00 & 5.00 & 6.00 & 10.00 & 8.00 & 10.00 & 9.00 & 7.00 & 4.00 & 8.00 \\
Total & 21.7 & 26.09 & 29.93 & 37.18 & 33.73 & 38.26 & 38.5 & 37.59 & 34.96 & 36.21 \\
\hline
\end{tabular}




\subsection{Analysis of the topic sequence}

During the course acquisition, learners were given the opportunity to use three topic sequences: teacher, learner, and optimal topic sequence, with the ability to change them after each topic. The results of the first and the second experiment showed that 41 students, or $64.1 \%$, used the topic sequence offered by the teacher (see Table 2). Only 23 students, or $35.9 \%$ of students, took the opportunity to choose a different topic sequence. Of these, 13 learners $(20.3 \%)$ used the optimal topic sequence and 10 students, $(15.6 \%)$ created their own learning path (learner topic sequence).

Table 2. Frequency of different topic sequences used in the first and second experiments.

\begin{tabular}{lll}
\hline Topic sequence & Frequency & Percentage $(\%)$ \\
\hline Teacher & 41 & 64,1 \\
Learner & 10 & 15,6 \\
Optimal & 13 & 20,3 \\
Total & 64 & 100,0 \\
\hline
\end{tabular}

The results show that most of the learners are passive in managing their own learning process or rely on the experience of the teacher in the sequence of topic acquisition and use the system's default topic sequence, i.e. the teacher topic sequence.

\subsection{Relationship between topic grades and topic sequence choice}

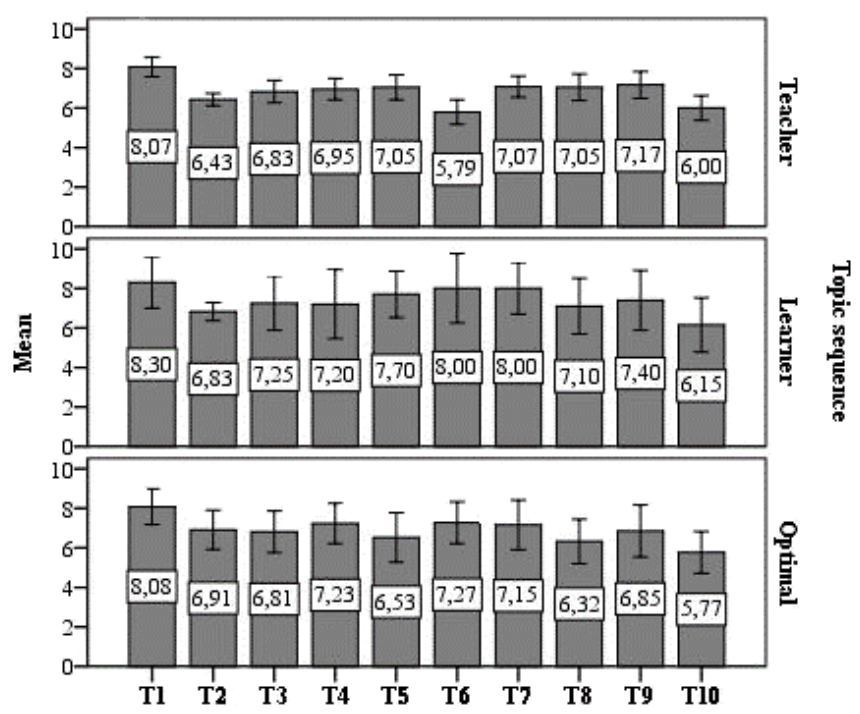

Figure 2. Arithmetic mean grades of each topic of the course "Programming Foundations I" depending on the selected course topic sequence 
The arithmetic mean (Mean) grade of each topic was calculated depending on the topic sequence used in the first and the second experiment (see Figure 2).

In the Figure 2, mean grades of different topics are divided into three sections depending on the topic sequence students used (Teacher, Learner, Optimal). Topics are marked with a "T" followed by an index of the topic. The grading scale varies from 0 (the lowest) to 10 (the highest).

To investigate the relationship between arithmetic mean topic grades and the selected topic sequence, each topic got assigned a relationship, which describes the grade depending on the student's chosen topic sequence. The "T" was used to denote the topic grades obtained by the learners using the teacher topic sequence. "L" denotes topic ratings obtained using the learner topic sequence and "O" - using the optimal topic sequence, see Table 3 .

Table 3. The relationship between the arithmetic mean grades of topics depending on the selected topic sequence.

\begin{tabular}{ccc}
\hline Topic number & $\begin{array}{c}\text { The relationship between } \\
\text { arithmetic mean of the topic grades }\end{array}$ & $\begin{array}{c}\text { The topic sequence with } \\
\text { the highest mean topic grade }\end{array}$ \\
\hline 1. & $\mathrm{~T}<\mathrm{O}<\mathrm{L}$ & $\mathrm{L}$ \\
2. & $\mathrm{~T}<\mathrm{L}<\mathrm{O}$ & $\mathrm{O}$ \\
3. & $\mathrm{O}<\mathrm{T}<\mathrm{L}$ & $\mathrm{L}$ \\
4. & $\mathrm{~T}<\mathrm{L}<\mathrm{O}$ & $\mathrm{O}$ \\
5. & $\mathrm{O}<\mathrm{T}<\mathrm{L}$ & $\mathrm{L}$ \\
6. & $\mathrm{~T}<\mathrm{O}<\mathrm{L}$ & $\mathrm{L}$ \\
7. & $\mathrm{~T}<\mathrm{O}<\mathrm{L}$ & $\mathrm{L}$ \\
8. & $\mathrm{O}<\mathrm{T}<\mathrm{L}$ & $\mathrm{L}$ \\
9. & $\mathrm{O}<\mathrm{T}<\mathrm{L}$ & $\mathrm{L}$ \\
10. & $\mathrm{O}<\mathrm{T}<\mathrm{L}$ & $\mathrm{L}$ \\
\hline
\end{tabular}

\subsection{Relationship between topic grades, importance and complexity}

In the second experiment, 17 learners rated each topic according to its complexity and importance in the course according to the Likert scale from 1-10. The teacher also assessed each topic according to the complexity and importance, see results in Table 1.

Arithmetic mean rating for the complexity and importance were calculated for each topic. The learner arithmetic mean grades in topics were analysed depending on the complexity of each topic (see Figure 3).

Learners consider the topics number "4", "6", "7", "8" and "10" to be the most complex topics (with ratings above mean). In Figure 3 the mean value in the figure is represented using a dashed line. The results are very close to the teacher's rating of complexity (see Table 1).

The differences between the learners' and the teacher's complexity ratings for each topic are as follows (the position of each number corresponds to the topic number): 1.77 , $2.59,0.77,0.82,0.05,0.91,2.41,3.09,4.95,0.82$. The largest difference in rating is for a topic number "9" (the difference is 4.95). The complexity ratings for 5 topics similar (with a difference less than 1). 
The first topic is considered simple by the learners and the mean grade of this topic is the highest compared to the other topics (see Figure 3). The teacher also rated this topic the least complex. The lowest grade is for topic number "10", which students also find the most difficult. Grades higher than mean are for topics number "4", "5", "7" un "9". To conclude, learners assess the topic complexity in a similar way as a teacher.

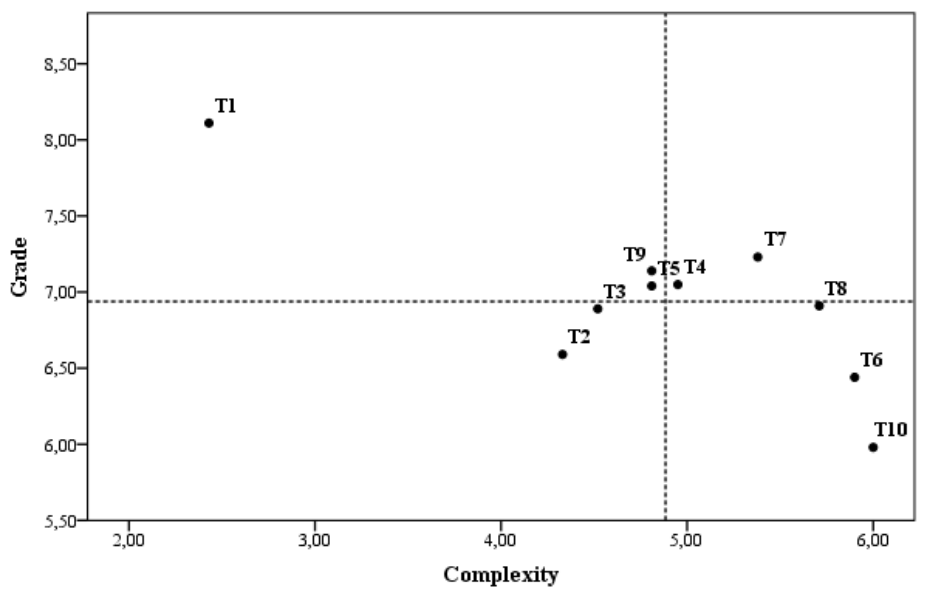

Figure 3. Relationship between the complexity rating and learner grades in each topic.

Analysing the importance and arithmetic mean grade of each topic (see Figure 4), it is concluded that students consider topics "2" and "3" less important than others. Other topics are considered more important by learners.

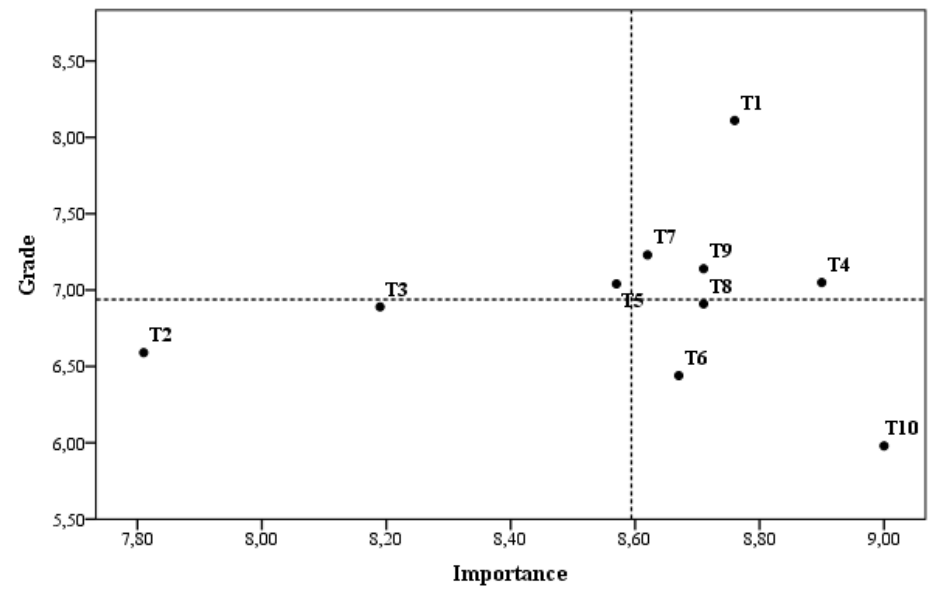

Figure 4. Relationship between the importance rating and learner grades in each topic. 
The differences between the learners' and the teacher's importance rating for each topic (see Table 1) are as follows (the position of each number corresponds to the topic number): $7.82,2.91,2.27,1.05,0.64,1.27,0.32,1.77,4.77,1.05$. The calculated differences show that the importance rating of the learners and the teacher are similar only for two topics. Small differences (less than 1) have topics number "5" and "7". The largest difference is for the topic "1" (the difference is 7.82). The topic importance rating of student and teacher differ much more than their complexity ratings. This can be explained by the lack of experience of the learner in the programming field. Out of the most important topics, the highest grades received topics "1", "4", "7", "8", and "9".

The analysis of the relationship between the arithmetic mean rating of complexity and importance of the topics showed how the learners perceive each topic of the course (see Figure 5).

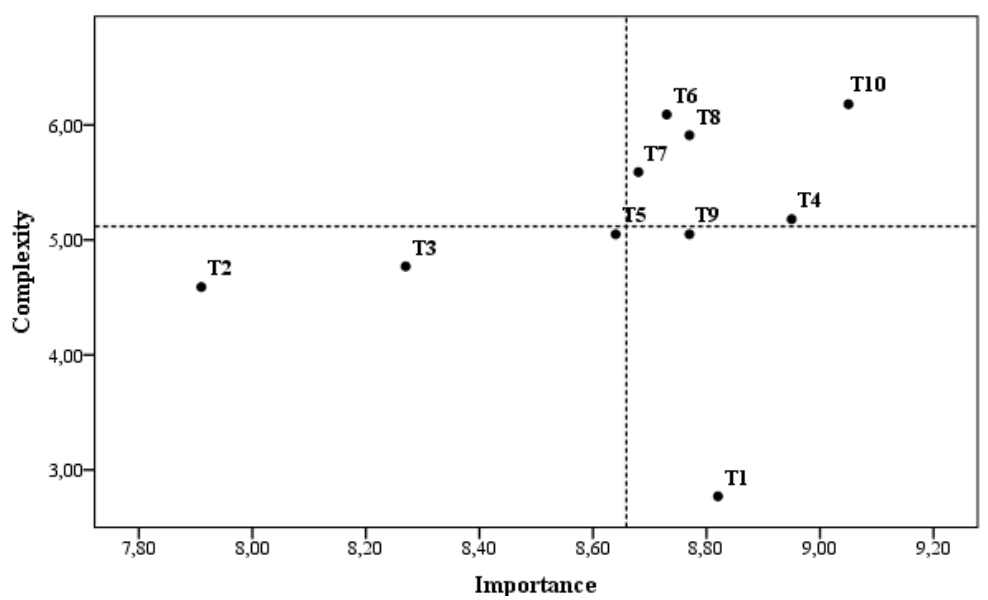

Figure 5. Relationship between the importance and complexity rating for each topic.

Students find topic "1" non-complex and important. Topics number "2" and "3" are non-complex and non-important. Topics number "5" and "9" have mean complexity ratings (the mean value is represented by a dashed line in the figure). Topic number "9" is considered important. The other topics are both important and complex.

\section{Improving the optimal topic sequence method}

This section describes the main results of the second stage of the study. Based on the $1^{\text {st }}$ stage results, an improvement of the optimal topic sequence creation method using the recommended learning path algorithm is proposed in this section. The recommended learning path algorithm has been validated with the help of an example. The recommended learning path of the course used in the study experiments is obtained.

\subsection{Definitions and assumptions}

A directed graph is used for the deeper examination of the course topics and links between them. A directed graph $G=(V, E)$ consists of a non-empty finite set $V$ of 
elements called vertices and a finite set $E$ of distinct ordered pairs of vertices called directed edges. We assume that course topics are vertices of the graph and the links between topics are the edges of the graph. Topics are described by a finite set $V=\left\{V_{l}, V_{2}, \ldots, V_{k}, \ldots, V_{n}\right\}$, where $\mathrm{n}$ is the number of topics in the course and the graph vertex index represents the order number of the topic in the course. For example, the vertex $V_{l}$ represents the first topic. Vertices $V_{i}$ and $V_{j}$ are connected using a directed edge where $V_{j}$ is the head and $V_{i}$ is the tail. This directed edge means that after finishing to study topic $V_{i}$, it is allowed to study topic $V_{j}$. The links between topic are defined by the developer of the course based on the acquired knowledge and competencies. Links between topics of the "Programming Foundations I" course that was used in the experiment are represented in Figure 1.

For getting a better description of the order of the topic in the course, each topic gets assigned parameters describing the topic, i.e. each vertex of the graph is described using a finite set of parameters. In the general case, $V_{k}=\left\{p_{1}, p_{2}, \ldots, p_{m}\right\}$, where $V_{k}$ is the graph vertex, $p_{l}, p_{2}, \ldots, p_{m}$ are the parameters and $m$ is the number of parameters. Every vertex is described using the same parameters. The weight of the vertex $V_{k}$ is $W V_{k}$ and it is the total sum of the vertex parameter values $p v$. See Formula 1, where $p v_{i}(1 \leq i \leq m)$ is the value of parameter and $m$ is the number of parameters.

$$
\mathrm{WV}_{k}=p v_{1}+p v_{2}+\cdots+p v_{m}
$$

The edge weight $W E_{i j}$ between two vertices $V_{i}$ and $V_{j}$ is the absolute difference of the weights of the both vertices (see Formula 2).

$$
W E_{i j}=\left|W V_{\mathrm{j}}-W V_{\mathrm{i}}\right|
$$

Smaller absolute difference between weights of two vertices indicates the smaller edge weight between the vertices. The edge weight describes how similar the two vertices are according to their parameter values. The development of the course recommended learning path is based on the search of the edge with the lowest weight. The direction of the edges between vertices are defined using adjacency vertices matrix. Adjacency vertices matrix $A$ is created as follows: if there is an edge from node $i$ to node $j$, then value " 1 " is entered in the corresponding row $i$, column $j$ of the matrix $A$.

\subsection{Algorithm of recommended learning path}

Finding a recommended learning path (RLP) is described using an algorithm. The following conditions were considered in the development of the RLP algorithm: (a) the course should always start with the first topic and (b) the course should include all the topics included in the course description (document describing the course requirements). The algorithm is based on 13 steps shown in the activity diagram in Figure 6. Initially (step 1-3), three arrays are initialised: adjacency vertices matrix $A$ (see Section 5.1), vertices weight array $W V$ (see Formula 1), and edges weight matrix $W E$ (see Formula 2). Next, the RLP development begins. The first vertex is recorded at the beginning of the $R L P$ (step 4).

In the weights matrix WE, the first (current) row that corresponds to the vertex recorded in the RLP is selected (step 5). The lowest number in the current row is searched next. The current row assumes the first number as the smallest (step 6). If the RLP has not yet recorded a vertex with the last sequence number, then the current row is 
searched for the smallest number given the existence of the edge between vertices (i.e. the values of matrix A) (step 7). If the RLP already has a vertex with the last sequence number, then the next smallest number is searched in the current row without considering the values of matrix A (step 8). This is possible because in RLP there is always a written topic with the number " 1 ", which means that there will always be a path from the first vertex to a vertex that is smaller than the last vertex. If the vertex with the last sequence number has not yet been recorded in the RLP, the position of the lowest number found (the vertex found) is recorded in the RLP (step 9), otherwise it is checked whether the vertex found is already in the RLP (step 10). If the found vertex is already written in the RLP, the smallest number found is saved (step 11) so that in step 8 the next smallest number can be searched for. If a vertex with the last sequence number was added, this event is saved (step 12). The algorithm stops working when all vertices of the graph have been added to the RLP. In step 13, the developed RLP is returned as a result.

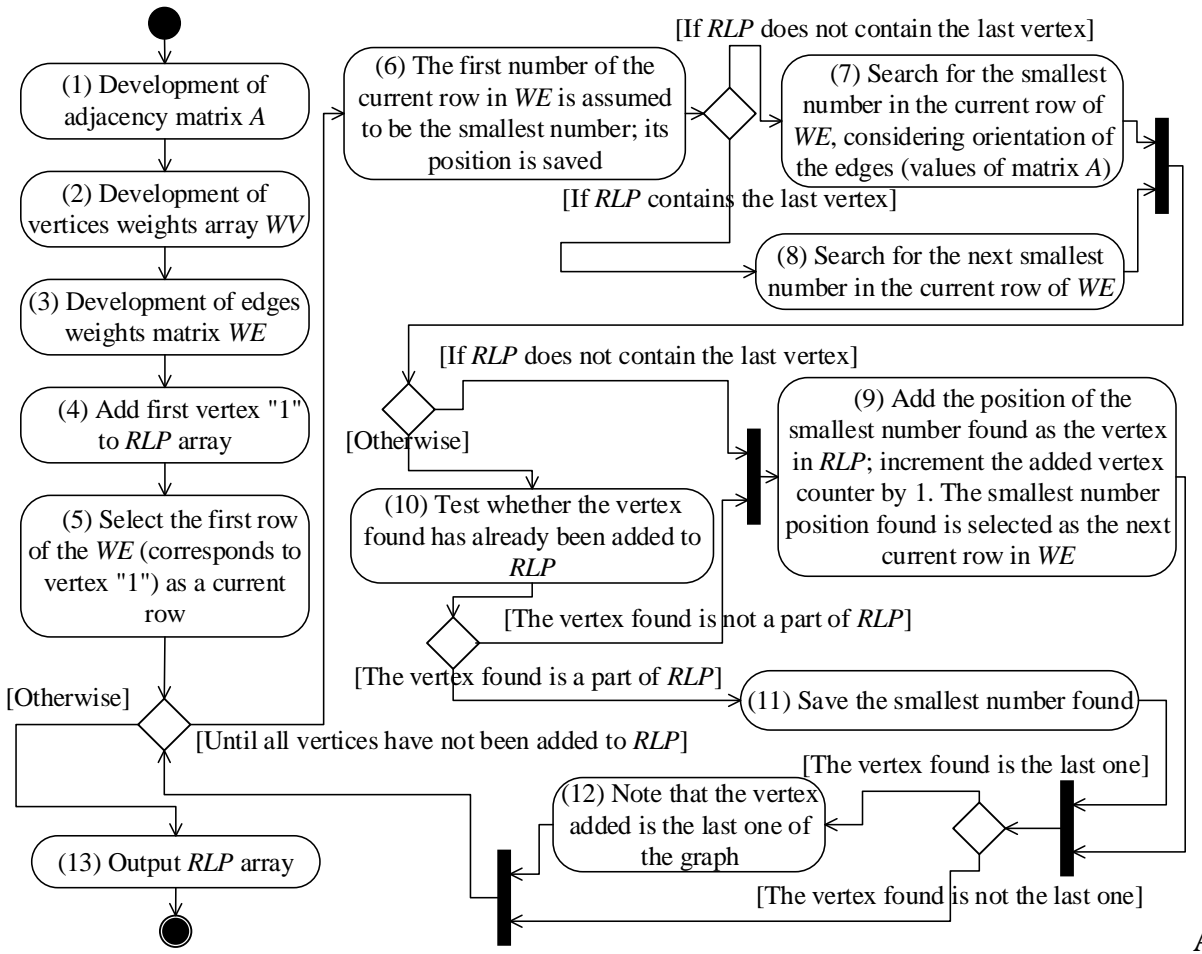

Figure 6. An activity diagram of the recommended learning path creation.

\subsection{Example of the recommended learning path search}

Using the algorithm described in Section 5.2, the recommended learning path for the "Programming Foundations I" course used in the experiment was searched. The structure of the course is described in Section 3.2. The links between the topics are shown in Figure 1. Each topic is described with the following five parameters: 
- $\mathrm{p} 1$ - the grade in the topic;

- $\mathrm{p} 2$ - the rating of the topic complexity according to the learner;

- $\mathrm{p} 3$ - the rating of the topic complexity according to the teacher;

- p4 - the rating of the topic importance according to the learner;

- $\mathrm{p} 5$ - the rating of the topic importance according to the teacher.

The acquisition of these parameter values is described in Section 4. The arithmetic mean values of the parameters obtained in the experiment are summarised in Table 1. The sum of the parameter values describing topics (using Formula 1) were assumed to be the weights of the graph (see Figure 1) vertices used in the recommended learning path search. Differences between the weights of each vertex were calculated (using Formula 2 ), i.e. edge weights with values summarized in Table 4 , where $V_{1}, V_{2}, \ldots, V_{10}$ are graph vertices.

Figure 7 shows a directed weighted graph where the numbers on edges represent the edge weight, or the absolute difference between vertex weights.

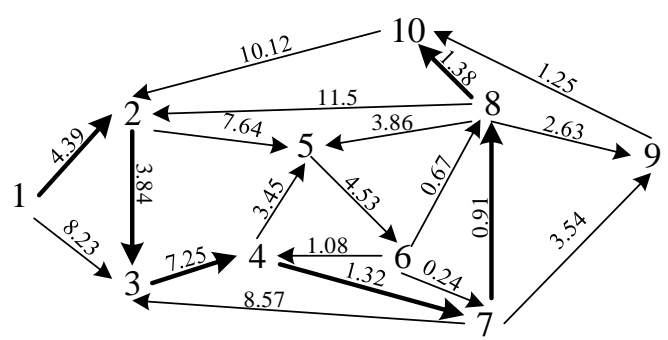

Figure 7. The graph of the course topic acquisition with weights on edges.

The vertices of the graph are represented as numbers that correspond to the course topic sequence numbers. While the vertex with the last sequence number has not been added to the RLP yet, the least weighted edges are searched starting from the first vertex, and the vertex number of the head of the directed edge is added to the RLP. The path

Table 4. Weights of the course topic graph edges.

\begin{tabular}{ccccccccccc}
\hline Weight of edges & $\mathrm{V}_{1}$ & $\mathrm{~V}_{2}$ & $\mathrm{~V}_{3}$ & $\mathrm{~V}_{4}$ & $\mathrm{~V}_{5}$ & $\mathrm{~V}_{6}$ & $\mathrm{~V}_{7}$ & $\mathrm{~V}_{8}$ & $\mathrm{~V}_{9}$ & $\mathrm{~V}_{10}$ \\
\hline $\mathrm{V}_{1}$ & 0 & 4.39 & 8.23 & 15.48 & 12.03 & 16.56 & 16.8 & 15.89 & 13.26 & 14.51 \\
$\mathrm{~V}_{2}$ & 4.39 & 0 & 3.84 & 11.09 & 7.64 & 12.17 & 12.41 & 11.5 & 8.87 & 10.12 \\
$\mathrm{~V}_{3}$ & 8.23 & 3.84 & 0 & 7.25 & 3.8 & 8.33 & 8.57 & 7.66 & 5.03 & 6.28 \\
$\mathrm{~V}_{4}$ & 15.48 & 11.09 & 7.25 & 0 & 3.45 & 1.08 & 1.32 & 0.41 & 2.22 & 0.97 \\
$\mathrm{~V}_{5}$ & 12.03 & 7.64 & 3.8 & 3.45 & 0 & 4.53 & 4.77 & 3.86 & 1.23 & 2.48 \\
$\mathrm{~V}_{6}$ & 16.56 & 12.17 & 8.33 & 1.08 & 4.53 & 0 & 0.24 & 0.67 & 3.3 & 2.05 \\
$\mathrm{~V}_{7}$ & 16.8 & 12.41 & 8.57 & 1.32 & 4.77 & 0.24 & 0 & 0.91 & 3.54 & 2.29 \\
$\mathrm{~V}_{8}$ & 15.89 & 11.5 & 7.66 & 0.41 & 3.86 & 0.67 & 0.91 & 0 & 2.63 & 1.38 \\
$\mathrm{~V}_{9}$ & 13.26 & 8.87 & 5.03 & 2.22 & 1.23 & 3.3 & 3.54 & 2.63 & 0 & 1.25 \\
$\mathrm{~V}_{10}$ & 14.51 & 10.12 & 6.28 & 0.97 & 2.48 & 2.05 & 2.29 & 1.38 & 1.25 & 0 \\
\hline
\end{tabular}


from the first topic to the last (tenth) with the smallest edge weight is shown in Figure 7 with bold lines. This means that $\operatorname{RLP}=\{1,2,3,4,7,8,10\}$. Some vertices were not considered along this path. Since the RLP contains the vertex with the last number, i.e. "10", then the next searched vertex is the vertex that has the smallest weight difference with the vertex "10" (see Table 4). Such a vertex is vertex "4". However, this vertex is already added to the RLP.

Then the vertex with the next smallest weight difference is taken. It is the vertex "9". The vertex found is added to the RLP, now $R L P=\{1,2,3,4,7,8,10,9\}$. Next, we search for the vertex with the smallest weight difference with vertex "9". Such a vertex is the vertex "5". Since this vertex is not yet added to the RLP, it is added now, so $R L P=\{1,2,3,4,7,8,10,9,5\}$. Next, we search for the vertex with the smallest weight difference with the vertex "5". Such a vertex is the vertex "7". However, it is already included in RLP. It is similar to the vertices "4", "3" and "8". Then the next vertex taken is "6". It has not been added to the RLP yet, so it is added now. As the result, $R L P=\{1,2,3,4,7,8,10,9,5,6\}$ and it includes all 10 vertices of the graph.

\section{The recommended learning path assessment}

The third experiment of the study was organised in Daugavpils University (Latvia) in the fall semester of the study year 2020/2021. During the study process, students were given the opportunity to take the course "Programming Foundations I" in the learner based personalized adaptive e-learning system LMPAELS. Three topic sequences were available in the course: the teacher, the learner and the recommended learning path topic sequence. The third experiment involved 16 first-year students of the professional bachelor study program "Information Technologies". At the end of the course, students took an adaptive course evaluation questionnaire. The experiment aimed to obtain data from the learning process to compare the topics grades in optimal topic sequence and recommended learning path cases. This was performed to test if there is an improvement in the level of grades.

\subsection{The use of topic sequences}

As a result of the third experiment, valid learning process data of 16 students were obtained. Firstly, it was analysed how many students used each one of the three topic sequences offered for the course. The results showed that 4 students $(25 \%)$ used the teacher topic sequence (see Table 5). 3 students (18.8\%) used the learner topic sequence and 9 students $(56.3 \%)$ used the recommended learning path.

Table 5. Frequency of the use of topic sequences in the course in the third experiment.

\begin{tabular}{lll}
\hline Topic sequences & Frequency & Percentage $(\%)$ \\
\hline Teacher & 4 & 25,0 \\
Learner & 3 & 18,8 \\
RLP & 9 & 56,3 \\
Total & 16 & 100,0 \\
\hline
\end{tabular}




\subsection{Analysis of topic grades}

The obtained topic grades in the case of optimal topic sequence (OTS) and recommended learning path (RLP) were compared. During the first and second experiments, the optimal topic sequence OTS $=\{1,3,5,7,8,9,10,2,4,6\}$ was used by 13 students (see Table 2). In the second stage of the study, the recommended learning path RLP $=\{1,2,3,4,7,8,10,9,5,6\}$ was developed for the course (see Section 5.2). In the third experiment, 9 students used the recommended learning path. Next, the arithmetic mean of topic grades was calculated both for optimal topic sequence and recommended learning path use, arithmetic mean of topic evaluations was calculated. Figure 8 shows the arithmetic means (Mean) of the grades obtained in each topic, both for the optimal topic sequence and the recommended learning path cases. In the figure, the topics are marked with the letter "T" followed by the topic sequence number.

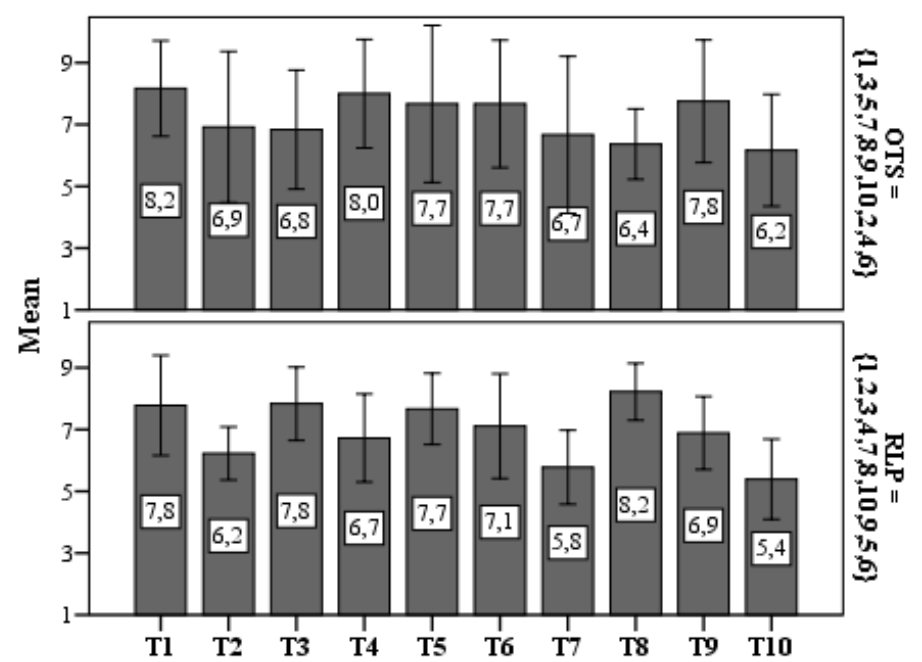

Figure 8. Arithmetic mean grades of each topic of the course "Programming Foundations I" in the optimal topic sequence and the recommended learning path cases.

The comparison of the obtained mean topic grades showed that in the case of using the optimal topic sequence the mean values are slightly higher than when using the recommended learning path. Only two topics "T3" and "T8" had higher arithmetic means $(\overline{\mathrm{x}})$ in the case of using the recommended learning path than the optimal topic sequence. The topic "T3" and "T8" had higher arithmetic means $(\bar{x})$ in the case of using the recommended learning path than the optimal topic sequence. The topic "T3" has $\overline{\mathrm{x}}_{\mathrm{OTS}}=6,8$ and un $\overline{\mathrm{x}}_{\mathrm{RLP}}=7,8$. The topic "T8" has $\overline{\mathrm{x}}_{\mathrm{OTS}}=6,4$ and $\overline{\mathrm{x}}_{\mathrm{RLP}}=8,2$ (see Figure 8).

An independent samples test was performed to determine the significance of the topic grade differences in OTS and RLP cases. The test results are summarized in Table 6. The left side of the table presents the Levene's Test for Equality of Variances results, which are used to test the uniformity of the sample variance.

Table 6 shows the F-statistic value $(F)$ of the test and the corresponding p-value of significance level p (Sig.). Levene's test results show that only one topic "T2" has a 
variance (root-mean-square deviation from the arithmetic mean) that differs significantly $(p=0,03<0,05)$. For the other nine topics, the sample variances do not differ significantly.

Next, the sample means were analysed with the help of t-test for Equality of Means. The test results are summarised on the right side of Table 6 , which shows the following values: t-statistic actual value (t), number of degrees of freedom (df), two-tailed alternative $\mathrm{p}$ value (Sig. (2-tailed)), sample mean difference, and a 95\% confidence interval of the difference. In this test, $\mathrm{p}$ values (Sig. (2 tailed)) were chosen depending on the Levene's test results. The results of the t-test for Equality of Means show that statistically significant grades (Sig. $(2$-tailed) $=0,009<0,5)$ are only for the topic "T8".

Table 6. Independent Samples Test results of topic mean grades.

\begin{tabular}{|c|c|c|c|c|c|c|c|c|}
\hline \multirow{3}{*}{ Topics } & \multicolumn{2}{|c|}{$\begin{array}{c}\text { Levene's Test for } \\
\text { Equality of Variances }\end{array}$} & \multicolumn{6}{|c|}{ t-test for Equality of Means } \\
\hline & \multirow[t]{2}{*}{$\mathrm{F}$} & \multirow[t]{2}{*}{ Sig. } & \multirow[t]{2}{*}{$\mathrm{t}$} & \multirow{2}{*}{ df } & \multirow{2}{*}{$\begin{array}{l}\text { Sig. (2- } \\
\text { tailed) }\end{array}$} & \multirow{2}{*}{$\begin{array}{c}\text { Mean } \\
\text { Difference }\end{array}$} & \multicolumn{2}{|c|}{$\begin{array}{l}95 \% \text { Confidence Interval } \\
\text { of the Difference }\end{array}$} \\
\hline & & & & & & & Lower & Upper \\
\hline $\mathrm{T} 1$ & 2,11 &, 17 & ,39 & 13 & ,702 & ,39 & $-1,76$ & 2,54 \\
\hline $\mathrm{T} 2$ & 6,12 &, 03 & ,78 & 13 &, 521 & ,69 & $-1,23$ & 2,62 \\
\hline $\mathrm{T} 3$ & ,41 &, 53 & $-1,14$ & 13 & ,274 & $-1,00$ & $-2,89$ & ,89 \\
\hline $\mathrm{T} 4$ & , 16 &, 70 & 1,36 & 13 & ,198 & 1,28 &,- 76 & 3,31 \\
\hline T5 & 3,03 &, 11 & ,00 & 13 & 1,000 &, 00 & $-2,17$ & 2,17 \\
\hline T6 & ,21 & ,66 & ,50 & 13 & ,627 & ,56 & $-1,85$ & 2,97 \\
\hline $\mathrm{T} 7$ & 2,54 & ,14 & ,87 & 13 & ,400 & ,89 & $-1,32$ & 3,10 \\
\hline $\mathrm{T} 8$ & 09 & ,76 & $-3,05$ & 13 & ,009 & $-1,86$ & $-3,18$ &,- 54 \\
\hline T9 & ,04 & ,85 & ,97 & 13 & ,349 & ,86 & $-1,05$ & 2,78 \\
\hline $\mathrm{T} 10$ & 03 &, 85 & ,87 & 13 & ,402 & ,78 & $-1,16$ & 2,72 \\
\hline
\end{tabular}

Further, in-depth research of topic grades was performed using descriptive statistics. The results of the analysis of topic grades can be seen in Table 7 when OTS was used, and in Table 8 when RLP was used.

Table 7. The statistics describing topic grades in the OTS case.

\begin{tabular}{|c|c|c|c|c|c|c|c|c|c|c|c|}
\hline & & $\mathrm{T} 1$ & $\mathrm{~T} 2$ & $\mathrm{~T} 3$ & $\mathrm{~T} 4$ & T5 & $\mathrm{T} 6$ & $\mathrm{~T} 7$ & T8 & T9 & $\mathrm{T} 10$ \\
\hline \multicolumn{2}{|l|}{ Mean } & 8,17 & 6,92 & 6,83 & 8,00 & 7,67 & 7,67 & 6,67 & 6,36 & 7,75 & 6,17 \\
\hline \multicolumn{2}{|c|}{ Std. Error of Mean } & ,60 & ,95 &, 75 & ,68 & ,99 &, 80 & ,99 & ,44 &, 77 &, 70 \\
\hline \multicolumn{2}{|l|}{ Median } & 8,50 & 6,75 & 7,00 & 8,50 & 8,00 & 7,00 & 7,00 & 6,84 & 8,00 & 6,00 \\
\hline \multicolumn{2}{|c|}{ Std. Deviation } & 1,47 & 2,33 & 1,83 & 1,67 & 2,42 & 1,97 & 2,42 & 1,09 & 1,89 & 1,72 \\
\hline \multicolumn{2}{|l|}{ Range } & 4,00 & 6,00 & 5,00 & 4,00 & 6,00 & 4,00 & 6,00 & 2,50 & 5,50 & 5,00 \\
\hline \multicolumn{2}{|l|}{ Minimum } & 6,00 & 4,00 & 4,00 & 6,00 & 4,00 & 6,00 & 4,00 & 5,00 & 4,50 & 4,00 \\
\hline \multicolumn{2}{|l|}{ Maximum } & 10,0 & 10,0 & 9,0 & 10,0 & 10,0 & 10,0 & 10,0 & 7,50 & 10,0 & 9,00 \\
\hline \multirow[t]{3}{*}{ Percentiles } & 25 & 6,75 & 4,75 & 5,50 & 6,00 & 5,50 & 6,00 & 4,00 & 5,00 & 6,38 & 4,75 \\
\hline & 50 & 8,50 & 6,75 & 7,00 & 8,50 & 8,00 & 7,00 & 7,00 & 6,84 & 8,00 & 6,00 \\
\hline & 75 & 9,25 & 9,25 & 8,25 & 9,25 & 10,0 & 10,0 & 8,50 & 7,13 & 9,25 & 7,50 \\
\hline
\end{tabular}


Statistically significant grades were obtained only for the topic "T8", so the statistical indicators characterising this topic are discussed below. In the case of OTS, the grades of the topic "T8" vary in relation to the mean value of 6,36 (Mean), in the range from 5,00 (Minimum) to 7,50 (Maximum) (see Table 7). The range of value variations is 2,5. The relative range of the variation is $2,5 / 6,36=0,39$. On average, the topic "T8" grades deviate from the mean by 1,09 (Std. Deviation). The coefficient of variation is $1,09 / 6,36=0,17$. The values of half of the "T8" topic grades do not exceed 6,84 (Percentiles, 50). 25\% of the grades are greater than $7,13.50 \%$ of the grades vary between 5 and 7,13 .

For RLP, the grades of the topic "T8" vary in relation to the mean value 8,22 (Mean) in the range from 6,00 (Minimum) to 10 (Maximum) (see Table 8). The range of value variations is 4,00 . The relative range of the variation is $4,00 / 8,22=0,49$. On average, the topic "T8" grades deviate from the mean by 1,20 (Std. Deviation). The coefficient of variation is $1,20 / 8,22=0,15$. The values of half of the "T8" topic grades, do not exceed 8,00 (Percentiles, 50). $25 \%$ of the grades are greater than $9,00.50 \%$ of the grades vary between 8,00 and 9,00 .

Table 8. The statistics describing topic grades in the RLP case.

\begin{tabular}{|c|c|c|c|c|c|c|c|c|c|c|c|}
\hline & & $\mathrm{T} 1$ & $\mathrm{~T} 2$ & $\mathrm{~T} 3$ & $\mathrm{~T} 4$ & T5 & T6 & $\mathrm{T} 7$ & T8 & T9 & $\mathrm{T} 10$ \\
\hline \multicolumn{2}{|l|}{ Mean } & 7,78 & 6,22 & 7,83 & 6,74 & 7,67 & 7,11 & 5,78 & 8,22 & 6,89 & 5,39 \\
\hline \multicolumn{2}{|c|}{ Std. Error of Mean } & ,70 & ,37 & ,51 & ,62 & ,50 & ,73 & ,52 & ,40 &, 51 &, 56 \\
\hline \multicolumn{2}{|l|}{ Median } & 8,00 & 6,00 & 8,00 & 6,00 & 8,00 & 8,00 & 6,00 & 8,00 & 7,00 & 5,00 \\
\hline \multicolumn{2}{|c|}{ Std. Deviation } & 2,11 & 1,12 & 1,54 & 1,86 & 1,50 & 2,20 & 1,56 & 1,20 & 1,54 & 1,69 \\
\hline \multicolumn{2}{|l|}{ Range } & 5,00 & 3,33 & 4,00 & 5,50 & 5,00 & 6,00 & 4,00 & 4,00 & 5,00 & 5,00 \\
\hline \multicolumn{2}{|l|}{ Minimum } & 5,00 & 4,67 & 6,00 & 4,50 & 5,00 & 4,00 & 4,00 & 6,00 & 4,00 & 4,00 \\
\hline \multicolumn{2}{|l|}{ Maximum } & 10,00 & 8,00 & 10,00 & 10,00 & 10,00 & 10,0 & 8,0 & 10,00 & 9,00 & 9,00 \\
\hline \multirow[t]{3}{*}{ Percentiles } & 25 & 5,50 & 5,34 & 6,00 & 5,50 & 6,50 & 5,00 & 4,00 & 8,00 & 6,00 & 4,00 \\
\hline & 50 & 8,00 & 6,00 & 8,00 & 6,00 & 8,00 & 8,00 & 6,00 & 8,00 & 7,00 & 5,00 \\
\hline & 75 & 10,0 & 7,25 & 9,25 & 8,50 & 8,50 & 9,00 & 7,00 & $\mathbf{9 , 0 0}$ & 8,00 & 6,25 \\
\hline
\end{tabular}

Since the means of the "T8" topic grades are different, the samples variation coefficients and the relative amplitudes of the variations are compared. In the case of OTS, the variation coefficient of topic "T8" is slightly higher than for RLP $(0,17>0,15)$. In the case of RLP, the relative amplitude of grades variation for the topic "T8" is slightly lower than for OTS $(0,49>0,39)$.

\subsection{Evaluation of the topic sequences from the students' perspective}

In all three experiments, an electronic survey was organised for students, after completing the course, to evaluate the adaptive course. This survey also offered to assess the truthfulness of the statement "The use of the topic sequence helped me to complete the study course in better quality" according to the Likert scale in the range from 1 (strongly disagree) to 5 (strongly agree).

Evaluation of the students' answers to this question concluded that the mean value of the answers that used RLP was slightly higher than that of the students who used OTS (see Figure 9). Evaluation of the statistical significance of the answers according to the results of the Independent Samples test concluded that the differences in the answers 
were not statistically significant, because the test results showed the value of statistical significance $\mathrm{p}=0,622>0,05$.

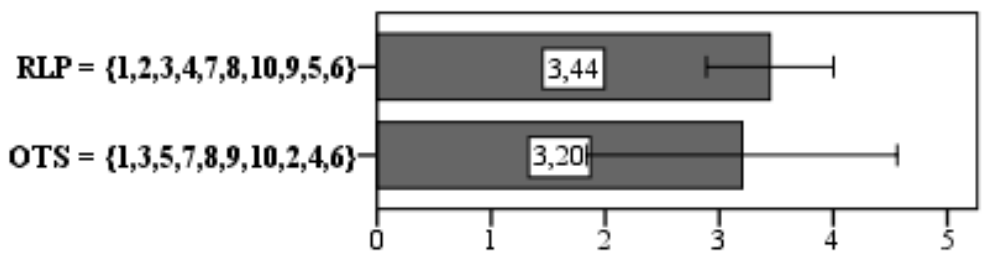

Figure 9. Evaluation of the statement "The use of the topic sequence helped me to complete the study course in better quality" for OTS and RLP.

\section{Conclusions}

The proposed study analysed how the use of the topic sequences offered by the personalized adaptive e-learning system influences the learning outcomes of the course. Three experiments were performed during the study. A total of 80 learners participated in these experiments. The sample size of the study is not large, so this have affected the significance of the study results.

The data obtained during the first experiment showed that, in general, learners are passive in organising their own learning process. Only 23 students, or $35.9 \%$ of the learners, took advantage of the system's option to use a topic sequence that is different from the teacher topic sequence. A small part of students, only 10 students $(15.6 \%)$, developed their own course topic sequence based on the links between the topics. These learners received the highest grades in eight topics. The obtained results could be explained both by the chosen topic sequence and the attitude of students towards the learning process. This assumption might be addressed in future studies.

The course used in the experiment was "Programming Foundations I" with OTS $=\{1,3,5,7,8,9,10,2,4,6\}$. This topic sequence was used by 13 students $(20.3 \%)$. Grades of students using OTS, in the topics, were lower than grades of students using LTS and higher than grades of students using TTS.

In this study, an algorithm for the development of a recommended learning path was developed that considers the characteristics of the topic. Additionally, the number of these parameters can be arbitrary. Using the developed algorithm for the "Programming Foundations I" course, the recommended learning path was created, $\mathrm{RLP}=\{1,2,3,4,7,8,10,9,5,6\}$. Parameters such as topic grades, complexity, and importance ratings were considered when designing the path.

In the third experiment, instead of using the optimal topic sequence, the obtained recommended learning path was used by 9 students. The results of the third experiment showed that, when using RLP instead of OTS, the grades of the course "Programming Foundations I" had improved for two topics - "T3" "Mathematical functions" and "T8" "One-dimensional numeric arrays". These topics are important both for studying the programming course and in other study courses related to programming (for example, in the course "Algorithms and Data Structures"). Only the grades of "T8" are statistically significant. This means that, in comparison with the use of OTS, the newly developed RLP helped students to better prepare for the topic "One-dimensional numeric arrays". 
As the study did not produce significant results due to the small sample size, this underlines the need to test the developed recommended learning path algorithm for a larger sample group in the future. Future work also includes continuing to study the use of student-centred approach in the personalized adaptive system.

\section{References}

Aeiad, E., Meziane, F. (2019). An adaptable and personalised E-learning system applied to computer science Programmes design, Education and Information Technologies 24, 14851509.

Attard, A., Di Iorio, E., Geven, K., Santa, R. (2010). Student-Centred Learning: Toolkit for Students, Staff and Higher Education Institutions, European Students' Union (NJ1), Laserline, Berlin.

Durand, G., Belacel, N., LaPlante, F. (2013). Graph theory based model for learning path recommendation, Information Sciences 251, 10-21.

Heberle, F., Henning, P. A., Streicher, A., Swertz, C., Bock, J., Zander, S. (2014). Advancement of MOOCs with learning pathways, International Journal of Excellence in Education 184(1792), 1-9.

Jurane-Bremane, A. (2018). Formative assessment in the study process, $\mathrm{PhD}$ thesis, University of Latvia, Riga, Latvia.

Lea, S. J., Stephenson, D., Troy, J. (2003). Higher Education Students' Attitudes to Studentcentred Learning: Beyond 'educational bulymia'?, Studies in Higher Education 28(3), 321334.

Niknam, M. (2017). LPR: an adaptive learning path recommendation system using ACO and meaningful learning theory, $\mathrm{PhD}$ thesis, The University of Manitoba, Manitoba, Canada.

Patel, N., Sellman C., Lomas, D. (2017). Mining frequent learning pathways from a large educational dataset, arXiv preprint arXiv:1705.11125, available at https://arxiv.org/pdf/1705.11125.pdf.

Vagale, V., Niedrite, L. (2014). Learner Classification for Providing Adaptability of E-Learning Systems, in Haav, H.-M., Kalja, A., Robal, T. (eds.), Proceedings of the 11th International Baltic Conference, Baltic DB\&IS 2014, TUT Press, Tallin, Estonia, pp. 181-192.

Vagale, V., Niedrite, L. (2016a). The Application of Optimal Topic Sequence in Adaptive eLearning Systems, in Arnicans G., Arnicane, V., Borzovs, J., Niedrite, L. (eds.), Communications in Computer and Information Science, Baltic DB\&IS 2016, Vol. 615, Springer, Heidelberg, pp. 352-365.

Vagale, V., Niedrite, L. (2016b). The Organization of Topics Sequence in Adaptive e-Learning Systems, Frontiers in Artificial Intelligence and Applications, Vol. 291, IOS Press, pp. 327340.

Vagale, V., Niedrite, L., Ignatjeva, S. (2018). The Architecture of the Personalized Adaptive ELearning System, in Lupeikiene, A., Matulevičius, R., Vasilecas, O. (eds.), CEUR Workshop Proceedings, Baltic DB\&IS 2018, Vol. 2158, pp. 114-123.

Vagale V., Niedrite L., Ignatjeva S. (2020a). Implementation of Personalized Adaptive E-leaming System, Baltic Journal of Modern Computing 8(2), 293-310.

Vagale V., Niedrite L., Ignatjeva S. (2020b). The use of the recommended learning path in the personalized adaptive e-learning system, Communications in Computer and Information Science, 1243 CCIS, pp. 280-294.

WEB (a) Standards and Guidelines for Quality Assurance in the European Higher Education Area (ESG), (2015), Brussels, Belgium, available at https://enqa.eu/wpcontent/uploads/2015/11/ESG_2015.pdf

Received November 29, 2020, accepted November 30, 2020 